\title{
La formación ciudadana en los estudiantes universitarios $^{\prime}$
}

\author{
Luis Alejandro Padilla Beltrán ${ }^{2}$ \\ José Eduardo Padilla Beltrán ${ }^{3}$
}

Wilmer Hernando Silva Carreño ${ }^{4}$

«... la educación... tiene que ser defendida como esfera pública vital por derecho propio, o sea, como esfera pública cuyas dimensiones morales y pedagógicas ayudan a renovar la vida cívica» (Giroux, 2003:361).

\section{Resumen}

Este artículo presenta la fundamentación teórica y conceptual para abordar la comprensión de las competencias ciudadanas, desde un horizonte crítico, en la formación de los estudiantes universitarios. Se hace necesario para esto, recoger de forma general la conceptualización de la sociedad, así como la formulación de las competencias necesarias que sirven como los referentes de la formación ciudadana de los estudiantes universitarios, y la forma como el Ministerio de Educación Nacional las considera desde la perspectiva de las competencias genéricas.

Palabras clave: Competencias ciudadanas, formación ciudadana, cultura ciudadana, perspectiva crítica.

\footnotetext{
1 El presente artículo de investigación hace parte del trabajo realizado por los investigadores en el marco del proyecto «competencias ciudadanas críticas en la formación de los estudiantes universitarios», UPN, 2010.
}

2 Abogado, Universidad Militar Nueva Granada; Especialista en Docencia Universitaria, Universidad Santo Tomás; Magíster en Educación, Universidad Pedagógica Nacional. Docente Asistente del Programa de Relaciones Internacionales y Estudios Políticos, Universidad Militar Nueva Granada. Correo: luis.padilla@unimilitar.edu.co

3 Licenciado en Matemáticas, Universidad Pedagógica Nacional; Especialista en Orientación Educativa, Universidad Manuela Beltrán; Magíster en Administración y Supervisión Educativa, Universidad Externado de Colombia; Doctor en Educación, Newport University. Docente Asociado, Director del Centro de Investigaciones de la Facultad de Estudios a Distancia y Director del Grupo PYDES de la Universidad Militar Nueva Granada. Correo: eduardo.padilla@unimilitar.edu.co 


\title{
Citizen formation in Colombian higher eduaction context
}

\begin{abstract}
Is presented in this article the theoretical and conceptual understanding to address citizenship competencies from a critical horizon in the training of university students. It is necessary for this, generally collect the conceptualization of citizenship and the development of citizenship competencies as reference of citizen education of college students and the way the Ministry of Education are considered from the perspective of generic skills.
\end{abstract}

Key words: Citizenship skills, citizenship, civic culture, critical perspective.

\section{Aproximación a la formación ciudadana}

Hoy en día el ideal de democracia no es solamente un tipo de régimen político en el que sea «el gobierno del pueblo y para el pueblo», sino que en el marco de los Estados modernos es en donde la soberanía realmente sea de los ciudadanos, quienes transfieren el poder a unos representantes de manera temporal y a través de elecciones; pero también es el conocimiento de instituciones que deben buscar la justicia como su fin; para que esto deje de ser una utopía, se hace necesaria la formación de ciudadanos conscientes, críticos y participativos. Esto conlleva el hacer referencia a la relación histórica entre educación y formación ciudadana.

\subsection{Evolución general del concepto de ciudadanía.}

Una primera aproximación formal al concepto de ciudadano puede encontrarse en la ética de Platón, desde donde se entrevé cómo los griegos formularon el paso de la barbarie a la civilización y crearon el concepto de ciudadano, que viene de civitas y que se refería al que vive en la ciudad. Platón (428348 a.C.), define la educación como un proceso de perfeccionamiento y embellecimiento del cuerpo y el alma; señalando además cómo la sociedad, 
el Estado y el hombre, no son nada sin educación. Este filósofo se encarga de destacar las tres funciones principales de la educación, a saber: la formación del ciudadano; la formación del hombre virtuoso; y la preparación para una profesión u oficio. Más adelante, la Revolución Francesa (1789-1799) marcó el final definitivo del absolutismo y dio a la luz a un nuevo régimen donde la ciudadanía, y en algunas ocasiones las masas populares, se convirtieron en la fuerza política que dominó a Francia y sirvió de ejemplo para la independencia de las colonias inglesas y posteriormente para las españolas en territorio norte, centro y suramericano.

Luego, al pasar del oscurantismo a la modernidad, los enciclopedistas comienzan a pensar y difundir el conocimiento que se tenía hasta ese momento por medio de las enciclopedias, gracias a los desarrollos tecnocientíficos como la imprenta. Así, empieza a tener sentido el conocimiento y la educación. Siendo la nobleza y la aristocracia los que se preocupan por buscar que sus hijos aprendan y se eduquen, mientras que el pueblo en general se mantiene en la ignorancia.

Ahora bien, el concepto de ciudadano llega a Colombia por medio del Virrey José Ezpeleta, quien le da a traducir un libro del francés al español a don Antonio Nariño, alcalde regidor de la ciudad de Santafé de Bogotá, el tercer tomo de la obra Historia de la revolución de 1789 y del establecimiento de una constitución en Francia, en cuyas páginas se transcribe el texto completo de la Declaración de los derechos del hombre y del ciudadano, hecha por la Asamblea Nacional de Francia el 4 de agosto de 1789, y que consta de 17 artículos y un preámbulo. Nariño extrae de esta manera los derechos del hombre, en donde surgen las palabras que marcarían a los granadinos del aquel entonces: libertad, fraternidad e igualdad, entre otras; y retoma los conceptos de Estado de Nicolás Maquiavelo y la división de los poderes públicos planteados por Montesquieu en su libro «El espíritu de las leyes» (1748).

Esto permite ir conceptualizando la importancia de la educación en las personas. En efecto, según Contreras, ésta se bifurca en dos amplios conceptos: la heteroeducación y la autoeducación. La primera consiste en el proceso educativo impuesto, en el que la persona es formada por la familia, la sociedad o el Estado, y el segundo, la autoeducación, se da cuando es la misma persona la que busca tomar el conocimiento y la información e integrarla a su saber. Lo ideal es que ambos lineamientos estén complementados para que la educación sea un proceso que dé a las personas los medios e instrumentos necesarios para la configuración de su formación y de esta manera se establezca el conocimiento como una verdadera herramienta para la vida.

En esto consiste el hecho humano de la educación, en la formación de la conciencia moral, en posibilitar la capacidad de cada persona para discernir 
entre lo que es el bien y lo que es el mal. Por tanto, se considera que el punto más importante del proceso educativo es formar en la voluntad de cada estudiante, la capacidad de tomar decisiones libres regidas por las normas y valores éticos y morales que como persona y ciudadano, pueda o deba tener; configurando de esta manera su comportamiento individual y colectivo.

Por esto, la Ley General de Educación de Colombia (Ministerio de Educación Nacional de Colombia, MEN, 1994: 17) precisa en su artículo primero que «la educación es un proceso de formación permanente, personal, cultural y social que se fundamenta en una concepción integral de la persona humana, de su dignidad, de sus derechos y de sus deberes». Adicionalmente la Ley 30 de 1992 consagra:

la Educación Superior,... despertará en los educandos un espíritu reflexivo, orientado al logro de la autonomía personal, en un marco de libertad de pensamiento y de pluralismo ideológico que tenga en cuenta la universalidad de saberes y la particularidad de las formas culturales existentes en el país. Por ello, la Educación Superior se desarrollará en un marco de libertades de enseñanza, de aprendizaje, de investigación y de cátedra (MEN, Ley 30 de 1992 Art. 4).

Es así como el proceso educativo se puede resumir básicamente en tres fases, a saber: la educación como desarrollo, donde el educador es quien impulsa los cambios en los conocimientos en el educando; la educación como disciplina, que surge cuando este desarrollo no se deja a su libre albedrío sino que se guía para controlarlo o estimularlo; y la educación como formación, donde el educador busca no sólo transmitir conocimientos y orientaciones a su discípulo, sino posibilitar su desarrollo de forma integral (Contreras, año). Sin embargo, es importante tener en cuenta que realmente la educación no tiene estas fases, sino que es un proceso simultáneo que integra las fases mencionadas, sin tener en cuenta esta división señalada.

Por otra parte, existen innumerables autores que han tratado de configurar el concepto de educación, y que en estos intentos han surgido muchas reflexiones con diferentes posturas, matices, formas y tipos de comprender la educación. Ortega y Gasset (1963) hace referencia a Kerschensteiner, quien dice que el fin general de la educación es educar a ciudadanos útiles que sirvan a los fines del Estado y de la humanidad. Pero Ortega y Gasset niega esto, habla de la formación del ciudadano como uno de los tantos fines de este proceso, haciendo referencia a todos los aspectos de la vida del individuo. Si se educa con la intención única de formar ciudadanos útiles a los fines del Estado, se forman individuos para el ayer.

Por esto, se considera válido lo que propone este pensador español, porque si se educa para los fines de cualquier Estado, se perdería la posibilidad de 
formar personas libres al servicio de la humanidad y pasarían a ser piezas de un sistema político o máquinas de producción en un sistema de globalización y mercado como el que se nos está imponiendo hoy en día. La educación para la ciudadanía por tanto, se puede orientar teniendo en cuenta tanto la concepción de «ciudadanía» y de «educación», como también el papel que se le concede al conocimiento y a la experiencia en la construcción de los saberes y las competencias necesarias para el ejercicio de la ciudadanía, las funciones que desempeñen los distintos agentes que intervienen en el proceso educativo y la permeabilidad de la escuela ante los cambios, problemáticas, retos y evolución de la sociedad.

Desde un análisis curricular, en unas ocasiones la educación para la ciudadanía se plasma en una materia que tiene diversas denominaciones; en otras es un enfoque transversal; unas veces aparece explícitamente en los programas, y otras está implícita en los objetivos educativos, como sucede hasta ahora en nuestro país. Un estudio comparativo sobre las orientaciones y prácticas de educación para la ciudadanía concluye que esta expresión es polisémica y ambigua, siendo algunos de sus significados: enseñanza formal de los conocimientos relativos a las instituciones nacionales e internacionales y a los derechos humanos; desarrollo del pensamiento crítico a partir de actividades tomadas de diversas corrientes como la «pedagogía de la cooperación»; desarrollo de competencias y actitudes para la ciudadanía, a través de una implicación mayor del alumnado en los problemas reales de su propia comunidad, la democratización de las relaciones en la clase o en el seno de la institución escolar.

Teniendo en cuenta la diversidad de tendencias, cabe destacar tres aproximaciones que van de un menor a un mayor grado de implicación personal y de compromiso con la transformación social, cada una de las cuales incluye a la anterior (Kerr, 2000), a saber:

- Educación sobre la ciudadanía (conocimiento y comprensión de la historia, las estructuras, los sistemas de gobierno, la vida política, etc.).

- Educación a través de la ciudadanía (participación activa en la escuela y en la comunidad local, reforzando los conocimientos).

- Educación para la ciudadanía (herramientas, conocimiento y comprensión, destrezas y actitudes, valores y normas para participar activamente en la sociedad, en la vida adulta), (Martínez, 2006: 15).

Por consiguiente, se puede evidenciar la necesidad de analizar la distinción que debe hacerse entre un ejercicio de la ciudadanía que puede llamarse activo y otro pasivo; de plantear la ineludible responsabilidad de la educación escolar en la formación de ciudadanos, que, sin embargo, no puede ser 
asumida en exclusiva por la escuela sino debe serlo por la sociedad en su conjunto; de considerarla una tarea compartida y comunitaria, porque son diversos los lugares en que se juega la Educación para la ciudadanía, por lo que se requiere de la construcción de una comunidad socio-educativa que pueda inducir un proceso de socialización congruente.

De ahí que la formación del hombre no puede abstraerse de la realidad social concreta, en la cual discurre la existencia humana y en especial la colombiana, no se puede prescindir, en una palabra, de la realidad del mundo actual. La tarea de educar a los hombres implica prepararlos para que sean capaces de asumir una actividad social, valiosa y fecunda, a través del desarrollo multifacético de su personalidad. En otras palabras, la formación para la vida no puede encerrarse en las categorías de la adaptación y el éxito material.

Miguel Martínez Martin, en el ensayo «Formación para la ciudadanía y la educación superior» (2006), plantea cómo la universidad es el lugar donde el futuro profesional aprende el conjunto de saberes para ejercer su profesión. Señala también que una de las funciones de la formación universitaria es de carácter ético, y que no puede entenderse una formación universitaria de calidad, que no incorpore de forma sistemática y rigurosa situaciones de aprendizaje ético y de formación ciudadana.

Así, se identifican tres dimensiones formativas en la función ética de la universidad en la sociedad actual: 1.- la formación deontológica relativa al ejercicio de las diferentes profesiones; 2.- la formación ciudadana y cívica de sus estudiantes; 3.- la formación humana, personal y social, que contribuya a la optimización ética y moral de las futuras y futuros titulados.

Por tanto, después de haber desarrollado algunos aspectos que se consideran válidos para el acercamiento al problema de la educación, que por demás es complejo, a continuación se intentará ver en contexto con la educación superior. Entendiendo esta como el «Proceso permanente que posibilita el desarrollo de las potencialidades del ser humano de una manera integral, se realiza con posterioridad a la educación media o secundaria y tiene por objeto el pleno desarrollo de los alumnos y su formación académica o profesional» (Ley 30 de 1992, art. 1).

Si bien es cierto, que en Colombia en las dos últimas décadas se ha venido trabajando para que dicha educación cumpla con los indicadores de calidad, que exige el ámbito de la globalización, con el sistema de la acreditación de alta calidad, es importante señalar que dentro de este ámbito la parte humanista exigida por la misma Ley 30 , ha tomado relevancia y hoy a la luz de estas exigencia se propende, desde la autonomía de cada institución, por desarrollar políticas en valores que incluyen la ciudadanía. 
Por esta razón, una formación universitaria de calidad no puede separarse de la formación ciudadana y para esto se esbozan tres razones: la primera, relacionada con el concepto actual de formación universitaria; la segunda, con el objetivo de cohesión social y no sólo de competitividad que debería tener como misión la universidad hoy; y la tercera, derivada de las investigaciones sobre desarrollo moral y aprendizaje ético (MEN, Ley 1188 de 2008). Como se observa, hay una gran preocupación investigativa a nivel universitario sobre el factor humanístico. Bien lo precisa el profesor J. Santisteban cuando plantea las relaciones entre la formación ciudadana y la enseñanza de las ciencias sociales, la geografía y de la historia.

En sus investigaciones, Santisteban hace referencia a las representaciones políticas de jóvenes españoles al finalizar la enseñanza obligatoria (16 años). También muestra la propuesta conceptual a partir de la cual se indagaron estas ideas. Esta propuesta conceptual constituye el marco desde el cual se interpretan estos resultados y se interviene en la práctica. Esta investigación pretendió establecer, a partir de la aplicación de una entrevista escrita de naturaleza cualitativa, categorías de análisis para el diagnóstico de las concepciones que coexisten en la comunidad escolar sobre formación ciudadana y participación democrática, entendiendo que la escuela es un espacio de construcción social y cultural en la que todos los estudiantes aprenden dinámicas de interacción claves para su desarrollo personal y colectivo.

\subsection{Ciudadanía y formación ciudadana}

De acuerdo con la historia, para los griegos los ciudadanos eran los hombres que vivían en la ciudad, diferentes a esclavos y siervos. Luego, más adelante con el nacimiento del concepto de Estado, toma valor el ciudadano identificado como la persona a las que el Estado le reconoce tal situación, estos ciudadanos poseían un conjunto de derechos y libertades individuales, de naturaleza civil (especialmente de carácter económico). Este concepto de ciudadanía se ha forjado desde el siglo XVIII, mediante un proceso acumulativo.

En el siglo XIX la ciudadanía adquirió un carácter predominantemente político. Los ciudadanos eran aquellos que gozaban de la plenitud de derechos políticos, aunque para el caso colombiano, por ejemplo, sólo podían votar los hombres y para esto, debían tener un nivel de educación y patrimonio, por lo que sólo un grupo muy reducido podía participar de este tipo de democracia, situación excluyente que aunque no es la misma hoy día, se da con diferentes matices.

En efecto, la construcción de la ciudadanía ha sido un proceso vinculado a la consolidación del denominado Estado-nación y al progresivo establecimiento, en este marco, de la democracia representativa. Ya se ha señalado, por ejemplo, cómo en Colombia en el siglo XVIII se excluían a las mujeres de 
la participación civil o ciudadana; situación que fue superada mediante el plebiscito de 1957, cuando se le dio la posibilidad a la mujer para que votara y a partir de ese momento participara en los procesos electorales siguientes. En el siglo XX la ciudadanía, en occidente, adquirió un nuevo contenido más social. Ser ciudadano era tener derecho a recibir educación y asistencia, prestaciones sociales diversas, servicios públicos subvencionados, salario reglamentario y protección laboral. En suma, se puede indicar que dicho concepto involucra un reconocimiento y respeto de los derechos humanos, económicos, sociales y culturales. Es así como la ciudadanía moderna en el siglo XXI es también un resultado del desarrollo económico y social que permite configurar el «Estado del Bienestar» ${ }^{5}$.

Así, hasta aquí se ha visto la dimensión política y social de la ciudadanía, cómo el ser humano no sólo es sujeto de derechos de las dos primeras generaciones (ciudadanía política y social). Es sobre todo miembro de la sociedad civil, parte de un conjunto de asociaciones no políticas ni económicas esenciales para su socialización y para el cotidiano desarrollo de su vida. La importancia de la sociedad civil no es poca, sobre todo si se tiene presente que el conjunto de procesos constitutivos de la ciudadanía no ha sido automático, ni derivado de un progreso moral ineludible, o del desarrollo inercial en las instituciones públicas o un efecto milagroso del mercado. La construcción de la ciudadanía ha sido el resultado de múltiples conflictos sociales, de confrontaciones de valores, de enfrentamientos políticos, como también de conflictos entre las propias instituciones del Estado.

Se entrevé que la ciudadanía se enfrenta a un doble desafío. Por una parte, hay factores que ponen en cuestionamiento los contenidos de la ciudadanía adquirida, por otra, nuevos fenómenos plantean la necesidad de ampliar los contenidos y renovar el concepto de ciudadanía como por ejemplo la globalización. Entre los factores que cuestionan la ciudadanía, los más importantes son los que resultan de la crisis de los Estados del Bienestar y del aumento persistente del desempleo. En los países europeos por ejemplo, una parte de la población (que tiende a crecer) pierde progresivamente la praxis de sus atributos ciudadanos: no vota, no tiene trabajo, vive en zonas marginales, se siente excluida de las instituciones, no está conectada con el progreso de las redes de comunicación, está «fuera» (Cortina, 2005).

Es importante destacar cómo el concepto contemporáneo de ciudadanía viene siendo estudiado por autores como Benito Martínez quien plantea que la ciudadanía y la educación es una tendencia válida que debe estar orientada a: «La promoción de una corriente de opinión favorable a la concepción de Ciudadanía, entre los diferentes sectores implicados en el ámbito educativo. Una revisión del currículum que integre esta perspectiva global de la 
ciudadanía de forma transversal. Una acción educativa en el aula que integre esta concepción en sus objetivos, contenidos y metodologías» (2005).

Por tanto, el concepto de ciudadanía ha evolucionado, y hoy involucra unos nuevos temas para el manejo de la ciudadanía tales como lo ambiental, lo digital, los temas y planteamientos de lo relativo al género, a lo étnico y cultural. Además, también podría implicar lo relativo a los medios de comunicación y la virtualidad, lo que ha llevado a que los ciudadanos ahora se expresen de diferente manera y realicen marchas y manifestaciones, realizando presión social para exigir cambios de postura sobre determinados temas por parte del Estado, como son las políticas públicas y locales, lo que hace más fuerte la descentralización y el fortalecimiento de lo municipal, por lo que se podría entrever que a corto plazo tenga que pensarse en el fortalecimiento de la ciudadanía desde lo local.

\section{La cultura en el contexto de la formación ciudadana}

Por cultura se entiende «el conjunto de modos de vida y costumbres, conocimiento y grado de desarrollo artístico, científico, industrial de una época y de un grupo social determinado» ${ }^{6}$. Este concepto debe estar integrado al actual de ciudadanía, ya que éste se encuentra consagrado en la Constitución como marco legal de los derechos humanos de primera, segunda y tercera generación. Aún más, como un status moral que corresponde a las responsabilidades y deberes y por ende a la identidad cultural de la persona, que como sujeto entra a pertenecer a una sociedad, a pesar, como se sabe, de desigualdades enormes en materia económica, social y por ende culturales. Además, con la movilidad que hoy tienen las personas que viajan de un país a otro sin mayor inconveniente, la ciudadanía ha de ser un vínculo de unión entre grupos sociales diversos, lo que hace más complejas las relaciones culturales hoy en día. En consecuencia, al hablar de cultura se debe referir el multiculturalismo identificado como la consecuencia de ésta, concerniente a fenómenos sociales que derivan de la difícil convivencia y/o coexistencia en un mismo espacio social de personas que se identifican con culturas diversas.

Estos conceptos están inmersos dentro de la ciudadanía en tanto la cultura constituye un elemento fundamental, frente al desarrollo de fenómenos como el apartheid o la xenofobia, entre otros; que hacen que sea complicada la promoción y la praxis del respeto por la diversidad cultural. En efecto, suele suceder que la cultura dominante va a querer imponerse sobre las pequeñas minorías, y éstas a su vez, van perdiendo sus características y se van asimilando a la predominante. 
Aún más, en muchos países, especialmente en aquellas naciones desarrolladas, las personas y los ciudadanos, se ven abocados a convivir con muchas culturas de diferentes países o regiones, como es el caso de España, donde debido al desarrollo demográfico y a una convivencia multicultural como la cristiana, la árabe y la judía, encontramos expresiones religiosas diversas; o en el caso de Colombia en donde ciudades como Bogotá, son el refugio en que se integran personas que provienen de todas las culturas y regiones del país. Tal es el caso, también de aquel país en donde se celebra un mundial de futbol o los juegos olímpicos, en tanto allí confluyen muchas culturas que deben ser respetadas durante el tiempo que duren dichos eventos. Esto evidencia la urgente tarea de generar procesos de comprensión, respeto y promoción de los derechos particulares de las culturas, y por tanto la defensa de la multiculturalidad como una forma esencial de la praxis ciudadana.

Por ello en nuestra Constitución Política, en el artículo 1, se determina que Colombia se constituye como una Nación multiétnica y multicultural; y en el artículo 68 se establece que «Los integrantes de los grupos étnicos tendrán derecho a una formación que respete y desarrolle su identidad cultural». Por lo tanto, el ser ciudadano involucra necesariamente esta dimensión de reconocimiento y respeto por los valores que tiene Colombia con la diversidad cultural que la compone. En este sentido, los procesos educativos constituyen un referente constitutivo en el desarrollo social equilibrado de una nación, es decir:

la Educación para la ciudadanía puede ser un modo de conciliar el pluralismo y la condición multicultural. Precisa, por ello, ser reformulada para incluir la diversidad cultural, de forma que no sea excluyente sino integradora, en una ciudadanía universal. La noción de ciudadanía, no se debe asociar a una identidad nacional o a un conjunto de rasgos culturales o biológicos, sino a una comunidad que comparte por igual un conjunto de derechos democráticos de participación y comunicación (Martínez, 2005, p. 17).

El concepto actual de ciudadano está ligado a la Constitución del Estado moderno. Es el Estado el que vincula ciudadanía con nacionalidad. El ciudadano es el sujeto político, el poseedor de un estatuto que le confiere, además de derechos civiles y sociales, los derechos de participación política. Se es ciudadano de un Estado, no en una ciudad. Se es ciudadano porque se posee una nacionalidad, regulada por un Estado y solamente vale este estatuto en el ámbito de ese Estado (Cortina, 2007).

Sin embargo, en la actualidad este concepto de ciudadanía no parece suficiente para integrar las nuevas demandas democráticas. Porque precisamente el concepto de ciudadano se refiere a una realidad dinámica. La ciudadanía se ha ido ampliando como resultado del desarrollo social y 
civil del Estado democrático. El estatuto de ciudadano en la segunda mitad del siglo XX es a la vez resultado del progreso civil (extensión hacia las mujeres y los jóvenes), social (Estado del Bienestar) y político (mecanismos de participación y representación más amplios y eficientes) (Cortina, 2007).

La ciudadanía europea formal, por ejemplo, consiste en el derecho a circular, residir y trabajar en cualquier país de la Unión Europea. En la práctica es un derecho limitado por el rol del Consejo Europeo y las legislaciones nacionales, que imponen condiciones de seguros sociales, disponibilidad de recursos económicos, titulación profesional, etc., así como el derecho a votar y a ser elegido en las elecciones locales y en las elecciones al Parlamento Europeo, una disposición importante, pero por ahora poco efectiva. En este es excepcional la presencia de un diputado elegido en un país distinto al suyo. En cuanto a las elecciones locales lo menos que puede decirse es que los gobiernos nacionales no han sido muy diligentes en tomar las medidas para hacer efectivo este derecho y cuando lo han hecho han impuesto restricciones (por ejemplo en Francia los extranjeros comunitarios pueden ser concejales pero no ocupar cargos ejecutivos como Alcalde, Teniente de Alcalde o «adjoint»).

Se puede por tanto referir cómo «la Unión Europea se basa en los principios de libertad, democracia, respeto a los derechos humanos y de las libertades fundamentales, así como del Estado de Derecho» (Cortina, 2007). En efecto, los ciudadanos europeos pueden recurrir ante el Tribunal de Justicia, en Luxemburgo, los actos de las instituciones que consideren contrarios a los derechos fundamentales. Si el Consejo Europeo constata la existencia de una violación grave y continuada de dichos principios por parte de un Estado miembro, puede sancionarlo con la suspensión de algunos de sus derechos, derecho de voto inclusive. Del mismo modo, la UE puede adoptar medidas necesarias para combatir «cualquier discriminación basada en razones de sexo, raza, origen étnico, religioso y creencias, discapacidad, edad u orientación sexual» (Cortina, 2007).

En este sentido, para el nacionalizado colombiano Jesús Martin Barbero, «lo que nuestras sociedades están reclamando al sistema educativo es que sea capaz de formar ciudadanos y que lo haga con visión de futuro, esto es, para los mapas profesionales y laborales que se avecinan. Se necesita una educación que no deje a los ciudadanos inermes frente a las poderosas estratagemas de que hoy disponen los medios masivos para camuflar sus intereses y disfrazarlos de opinión pública».

Adicionalmente para Colombia, en un ensayo presentado por Sánchez Fontalvo se entrevé cómo la ciudadanía que necesita Colombia debe comprender cuatro modelos de la misma: ciudadanía multicultural, ciudadanía intercultural, ciudadanía democrática y ciudadanía democrática 
radical (2008). Esto con el fin de poder alcanzar un modelo de sociedad democrática, pluralista, intercultural y equitativa en Colombia. Además, y como se puede ver en los postulados de la Constitución del 1991, el Estado es participativo y pluralista, lo cual hace que estos imperativos deban ser tenidos en cuenta en las instituciones de educación superior, para formar en los estudiantes esa cultura ciudadana que tanto necesita el país.

\section{Competencias ciudadanas: concepciones y desarrollo}

\section{1. ¿Que es una competencia?}

La competencia se debe entender como un saber hacer con conciencia y en contexto. Es un saber en acción. Un saber cuyo sentido inmediato no es solamente «describir» la realidad, sino también buscar «modificarla»; no solo poder comprender los problemas, sino también buscarle las soluciones más adecuadas y aplicarlas; un saber del qué, pero también un saber del cómo. Las competencias son, por tanto, conocimientos que adquieren las personas en permanente modificación, que se van adquiriendo a lo largo de la vida a través del conocimiento y la experiencia, que le van a ayudar al estudiante y luego al profesional a resolver problemas concretos en diversas situaciones de su vida diaria y en especial en el trabajo. Por lo tanto las competencias son diferentes en cada situación y momento, lo que obliga a la persona a tener en cuenta su contexto en cuanto a tiempo, modo y lugar.

Las competencias se desarrollan a través de experiencias de aprendizaje en cuyo campo de conocimiento se integran tres tipos de saberes: conceptual (saber conocer), procedimental (saber hacer) y actitudinal (saber ser). Son aprendizajes integradores que involucran la reflexión sobre el propio proceso de aprendizaje (metacognición) (Pinto, 1999). Este conocimiento necesario para la resolución de problemas no es mecánicamente transmisible. Algunos autores lo llaman conocimiento indefinible, por tratarse de una mezcla de conocimientos tecnológicos previos y de experiencias concretas que proviene fundamentalmente del trabajo y del mundo real. La gran diferencia de este enfoque, con respecto a la escuela tradicional, es que la competencia no proviene solamente de la aprobación de un currículo basado en objetivos cognitivos, sino de la aplicación de conocimientos en circunstancias prácticas.

\subsection{Una tipología de las competencias}

Es importante tomar como punto de partida los pilares de la educación que plantea la Unesco (1998) que son: Aprender a conocer: concertar entre una cultura general suficientemente amplia y los conocimientos particulares de las diferentes disciplinas, en torno a problemas e interrogantes concretos. 
Esto requiere aprender a aprender, con el fin de aprovechar las posibilidades que ofrece la educación a lo largo de la vida. Aprender a hacer: adquirir no sólo una certificación profesional, sino más bien competencias que capaciten al individuo para hacer frente a gran número de situaciones previstas e imprevistas y a trabajar en equipo. Aprender a vivir juntos: realizar proyectos comunes y prepararse para asumir y resolver los conflictos, respetando los valores del pluralismo, el entendimiento mutuo y la paz, a través de la comprensión del otro y de las formas de interdependencia. Aprender a ser: actuar con creciente capacidad de autonomía, de juicio y responsabilidad personal, para que florezca en mejor forma la propia personalidad. Con tal fin, no se debe subestimar ninguna posibilidad de cada persona en su proceso educativo: competencias intelectuales (memorizar, razonar, comprender, etc.), comunicativas, afectivas, estéticas y físicas, entre otras.

Adicionalmente a estos cuatro pilares, la Unesco (Delors, 1966) complementa la especificación de las competencias universitarias de los egresados, de las competencias propias de cada profesión, integrándolas con los conocimientos y con las competencias cognoscitivas, comunicativas y socio afectivas.

Para el caso colombiano, el Ministerio de Educación Nacional recientemente amplió el concepto de competencias genéricas, que se entendían hasta ese momento, como las que se relacionan con los comportamientos y actitudes en labores propias de los diferentes ámbitos de producción (Larrain y González, s.f.). Ejemplo: capacidad para trabajar en equipo; saber planificar, habilidad para negociar, socializar, etc; en cambio hoy en día, el MEN cuenta con que éstas competencias genéricas busquen establecer referentes comunes, que garanticen la calidad de la formación de los futuros profesionales en Colombia y la articulación de los niveles educativos (inicial, básica, media y superior), para ello ha formalizado la identificación de cuatro competencias genéricas para la educación superior, a saber: comunicación en lengua materna y otra lengua internacional; pensamiento matemático; ciudadanía; ciencia, tecnología y manejo de la información.

Con el presente estudio se particulariza en la formación de la competencia ciudadana, donde se fomenta la autonomía y el pensamiento crítico en los estudiantes universitarios, de tal manera que se eduque para fortalecer la democracia, con un conocimiento e información que le brinde el sustento argumentativo, para el debate sobre las políticas públicas y demás actuaciones del gobierno de turno y adicionalmente pueda participar como debe ser en las elecciones, teniendo en cuenta las fuentes de la información y la toma de decisiones.

\section{3. ¿Qué es una competencia ciudadana?}

Las Competencias Ciudadanas tienen un fundamento social y filosófico, pero tienen también una significación más profunda, que consiste, para el 
estudiante, en el «saber hacer» con esos conocimientos; en saber comportarse como ciudadano informado y participativo. Por lo que la formación ciudadana debe ser un complemento del conocimiento disciplinar que se debe impartir a todos los estudiantes sin ser un saber más importante que el otro a todos los niveles educativos.

Esta formación debe abarcar habilidades y actitudes para la acción y la participación democrática, la asociación, la organización, la acción colectiva, el intercambio de opiniones, la expresión de puntos de vista, el respeto por la forma de pensar diferente y la modificación de posturas, entre otras. Además, tiene por fin último conseguir el «empoderamiento» del estudiante. En palabras de Antanas Mockus, «un buen ciudadano, un ciudadano competente, es quien sabe y tiene un conjunto de habilidades, conocimientos, disposiciones y actitudes favorables al desarrollo de la ciudadanía, que facilitan y propician su propia participación como ciudadano y también, los procesos colectivos de construcción de ciudadanía» (Mockus, 2004).

Por este orden de ideas, las competencias ciudadanas son un conjunto de conocimientos, habilidades y disposiciones para construir convivencia, participar democráticamente y valorar el pluralismo en la búsqueda del bien común (MEN, 2004). Se necesita pasar de una concepción que únicamente desarrolla ciudadanía pasiva a otra que, además, desarrolle una ciudadanía activa; esto es, que no solamente busque la formación en conocimiento, comprensión y conductas que permitan la convivencia en comunidad y la observancia de la ley, sino que además promueva el desarrollo de habilidades de participación para asumir posiciones críticas, debatir con argumentos sólidos y proponer modelos alternativos de estructuras y procesos democráticos, para construir nación y fortalecer de esta manera cada más la democracia sin la intervención de la violencia.

Para respaldar las consideraciones anteriores es necesario atender tres aspectos de la formación en ciudadanía ${ }^{7}$. El primero tiene que ver con la Educación sobre ciudadanía: suministrar a los estudiantes conocimientos y elementos suficientes sobre la historia nacional, los orígenes de la violencia con sus causas y consecuencias, las estructuras de los organismos que conforman el Estado y la relación gobierno - poder para entender cómo se desarrollan los procesos tanto del gobierno como de la vida política; además, propiciar el respeto por las diferencias y los procesos de convivencia.

El segundo tiene que ver con la Educación mediante el ejercicio de la ciudadanía: facilitar el aprendizaje mediante la participación activa de los estudiantes en experiencias democráticas reales dentro de la universidad, en el entorno inmediato o en la comunidad (por ejemplo, intervención en el 
gobierno estudiantil, en la realización de encuestas de opinión, en proyectos ambientales del colegio o de la comunidad). Este tipo de participación refuerza el componente de conocimiento, mediante la acción. El tercero hace referencia a la Educación para la Ciudadanía: formar para que los estudiantes cuenten con un conjunto de herramientas tales como conocimiento y comprensión; habilidades y aptitudes; valores y disposición a la acción, respeto y tolerancia que les permitan asumir de manera activa, sensata y crítica, cargos y responsabilidades a lo largo de toda su vida.

Pero además de las tres consideraciones previas, los estudiantes van a ejercer también la ciudadanía en un mundo cada vez más globalizado en todos los aspectos, principalmente en lo económico, en lo laboral, en lo social, en lo político, en lo cultural, etc.; lo que conlleva afrontar problemas globales que requieren visiones con soluciones igualmente globales. En efecto, ya un estudiante no puede apartarse de que vive en una aldea global, y el profesor no puede desconocer esto en la formación que le está impartiendo al estudiante y no tener en cuenta este aspecto tan importante en la vida educativa actual.

Con base en los tres aspectos señalados, los estudiantes tendrían así, la capacidad de ejercer la ciudadanía y de actuar con base en los principios concertados por una sociedad y validados universalmente. Además de relacionarse con la actuación de un individuo, las competencias ciudadanas implican la capacidad para efectuar juicios morales, conocer el funcionamiento del Estado y comportarse e interactuar con otros y consigo mismo. El desarrollo de estas competencias permite que los estudiantes participen activamente no sólo en la institución educativa, sino también en la esfera pública y en las organizaciones a las que se vinculen, para promover intereses colectivos, defender derechos y cumplir deberes como ciudadanos y miembros de una comunidad o grupo. Igualmente, les posibilita la reflexión y la crítica frente a su comportamiento y el de los demás, el manejo de conflictos y la asunción de posiciones argumentadas sobre los hechos importantes de la vida local, regional, nacional e internacional.

La formación de competencias ciudadanas está relacionada con la apropiación de mecanismos de regulación del comportamiento, tales como la ley, principios, valores, normas y reglamentos, creados para convivir en armonía con otros, que son diversos, así como para regular los acuerdos y respetarlos. En Colombia, los objetivos que se pretenden en primera instancia con las competencias ciudadanas, es que los estudiantes tengan la capacidad para aplicar conocimientos y habilidades cognitivas emocionales y comunicativas que lleven a: la convivencia pacífica; la participación y responsabilidad democrática; la pluralidad, identidad y valoración de las diferencias; todo esto enmarcado en el respeto y la defensa de los derechos humanos.

Con respecto a esto, las competencias ciudadanas por un lado deben favorecer la convivencia pacífica, la participación democrática con un reconocimiento 
de la diversidad étnica y cultural; y por el otro el conocimiento de la Constitución, su respeto y apropiación en la medida en que esta se haga viva. Además, para Keneth Dodge (1990) citado por Silva y Chaux, otra competencia que es fundamental para la convivencia social y que de no desarrollarse en los estudiantes, genera comportamientos agresivos es la «capacidad para interpretar adecuadamente las intenciones de los demás». La falta de ésta impide lograr una verdadera interacción social, lo que lleva a que piensen que les van a hacer daño, cuando en realidad las situaciones pueden presentarse como accidentes.

Estos autores presentan, además, cómo esta competencia sirve para que los estudiantes puedan «imaginarse distintas maneras de resolver un conflicto o una problemática social», que de no desarrollarse adecuadamente lleva a el problema que se presenta en Colombia en que la agresión y la muerte son maneras fáciles y rápidas de resolver conflictos. Esta competencia permite adicionalmente desarrollar en el estudiante la capacidad de evaluar de forma objetiva las opciones de las cuales dispone y con ello poder evaluar las consecuencias de cada una. Así, el pensamiento crítico es una de las competencias fundamentales para un verdadero ejercicio de la ciudadanía.

Un ejemplo de lo anterior, es que si los estudiantes comprenden realmente el contexto colombiano, pueden entender mejor el porqué del conflicto armado que vive Colombia desde hace 200 años y por supuesto plantear soluciones creativas desde su quehacer disciplinar. Aún más, las competencias comunicativas según Habermas, citado por Ruiz y Chaux (2001:65), constituyen «la capacidad de conocimiento, lenguaje y acción que se encuentran en la base de toda competencia, se forman en un enfrentamiento, a la vez constructivo y adaptativo del sujeto con su entorno". En efecto, la competencia comunicativa entendida como la capacidad de generar acción a través del lenguaje nos lleva a que no solo se comunican contenidos sino también el sentido en el que se usan dichos contenidos comunicados (Ruiz y Chaux, 2001:173).

Esto implica que con el desarrollo de esta competencia los estudiantes pueden y deben revisar la argumentación de las afirmaciones que les son comunicadas, en situaciones conflictivas pueden hacer conocer sus derechos y sus puntos de vista sin incomodar a los demás, y con ello expresar sus puntos de vista sin molestarse. Por esto es esencial para Colombia una ciudadanía con en este tipo de competencias, y se debe fomentar para lograr en algún momento desarmar la violencia de nuestro lenguaje y nuestra acción. Ahora bien, para el desarrollo de las competencias comunicativas, el estudiante debe desarrollar la competencia de saber escuchar a los demás, no solamente poniendo toda la atención a lo que le hablan, sino también, dándole a conocer a su interlocutor que está siendo escuchado; y esto lo puede hacer valorando los desacuerdos, estableciendo compromisos y cumpliéndolos. 
Por otra parte, es necesario llevar a la praxis cotidiana las competencias emocionales, entendidas estas como las capacidades necesarias para que una persona pueda identificar las emociones propias y las de los otros, y responder tanto a las unas como a las otras en forma adecuada y constructiva. En efecto, el conocer las propias emociones, le permite al ciudadano que cuando se encuentre de mal genio se pueda conocer y controlar para no hacerse daño y no hacerle daño a nadie, lo que lleva a que conozca las estrategias de autocontrol teniendo en cuenta para ello que no pueden ser las mismas estrategias para todas las personas. Por esto, otra característica de las competencias emocionales seria la empatía (Ruiz y Chaux, 2001: 42), es decir, la empatía es fundamental para la convivencia en sociedad, ya que es la capacidad de sentir algo parecido o compatible con lo que puedan estar sintiendo otras personas. Para Martin Hoffman (2002) citado también por los autores Ruiz y Chaux, la empatía contribuye a que las personas se preocupen por ayudar a quienes lo necesitan, eviten herir a otras física y sicológicamente o busquen el perdón y la reconciliación con otros cuando se dan cuenta que han hecho daño.

De ahí que un caso particular como Colombia, donde a partir del año 1997 se han desplazado747.719 familias, para un total de 3.292.667 personas, según datos del gobierno (aunque para los organismos no gubernamentales la cifra supera los fácilmente los 4.2 millones de desplazados $)^{8}$, exige que al menos este fenómeno del desplazamiento, se aborde con una postura crítica y propositiva con respecto a la ciudadanía, ya que es ésta la que se desplaza o se hace indiferente frente al fenómeno. Por ello es que es importante que para Colombia, en la formación de ciudadanos, se tenga en cuenta esta variante de la competencia emocional.

De forma conjunta, si la ciudadanía como concepto político propone que todos los seres humanos son iguales, que todos tienen derecho a tener derechos, que se es ciudadano en tanto que se actúa en la sociedad a partir de reglas equitativas y justas de convivencia; la ciudadanía dentro del proyecto de construcción de sociedad democrática, implica también «la adquisición de referentes y competencias políticas capaces de propiciar y estimular la participación activa en los espacios políticos. Obviamente se trata de ir más allá de participar en las elecciones y votar. Se trata de adquirir información, desplegar intereses, cultivar actitudes y adoptar criterios que contribuyan a construir y enriquecer un espacio público plural» (Cabrera, 2002).

La ciudadanía es al mismo tiempo una aventura para dejar la condición de individuo como ser particular y ganar la experiencia de lo colectivo como ser social. La ciudadanía es aún más importante, en tiempos en que se asiste a una crisis de los agentes tradicionales de socialización (iglesia, familia, escuela); 
una crisis de las formas de representación (partidos políticos, sindicatos); una efervescencia de un sistema político llamado democracia (un adjetivo, más que un ethos); una moda de movilización social-territorial (una combinación entre arribismo y desplazados). Con todo, en la educación, se ha ido sustituyendo el concepto de educación cívica por el de competencias ciudadanas.

Por esto, el concepto que se asume en este trabajo con respecto a las competencias ciudadanas es el del Ministerio de Educación Nacional, que considera que éstas son los «conocimientos y habilidades cognitivas, emocionales y comunicativas que hacen posible que las personas participen en la construcción de una sociedad democrática, pacífica e incluyente» (MEN, 2002). Por ejemplo, el conocimiento sobre los mecanismos constitucionales para proteger los derechos fundamentales, como la tutela, es importante para participar democráticamente y para hacer que nuestros derechos sean respetados. La capacidad para imaginar distintas alternativas creativas de solución es una competencia cognitiva para poder resolver pacíficamente conflictos entre personas o entre grupos.

El reconocimiento y manejo de las emociones propias, es una competencia emocional fundamental para relacionarse pacíficamente con los demás. Por ejemplo, si soy capaz de mantener cierto control sobre mi ira en situaciones estresantes, es más fácil que pueda evitar hacerles daño a otros o a mí mismo en esas situaciones. La capacidad para escuchar seriamente los puntos de vista de los demás, así sean contrarios a los propios, es un ejemplo de competencia comunicativa fundamental para vivir en una sociedad donde todos tienen derechos y obligaciones ${ }^{9}$.

Conocer cómo funciona el Estado y cuáles son los derechos humanos permite que los estudiantes los asuman y caractericen su sentido. Para esto el concepto de Estado Social de Derecho, cuando es comprendido, se entiende que hay unos mínimos de justicia, paz y libertad que deben ser respetados si el Estado pretende ser considerado como legítimo; debido a esto es que se debe entender que el Estado debe «incluir en el sistema de derechos fundamentales, no solo las libertades clásicas sino también los derechos económicos, sociales y culturales» (Cortina, 2005: 77).

Ahora, en un mundo global como el que está emergiendo, se está creando el concepto de la sociedad digital, esto es, se han creado nuevas formas de alfabetización que no se pueden dejar de lado por lo que la formación ciudadana también implica ser competente en el mundo digital. El acceso a la información globalizada, los sistemas de participación en la red, las redes sociales, la comunicación a través de los medios electrónicos, son elementos de importancia para el desarrollo de competencias ciudadanas, porque de lo 
contrario se estaría frente a una nueva acepción de la palabra analfabetismo que se podría definir como el analfabetismo digital.

\section{Desarrollo de competencias ciudadanas desde la perspectiva crítica}

El concepto de ciudadanía crítica que se trabajó en la presente investigación corresponde a un proceso en formación permanente de derechos y responsabilidades personales y sociales, puestas en ejercicio en proyectos colectivos de bienestar común, construidos desde la diferencia y la multiculturalidad, con respeto a la alteridad y tolerancia a las diferencias, con un compromiso claro de cambio de nuestra desigualdades y divisiones. Implica una ciudadanía respetuosa de la diferencia y denunciadora de cualquier tipo de discriminación, sin violencia; fundamentada en el respeto, la justicia, la solidaridad y la participación. Entendiendo ésta, la participación, por un lado como el derecho a elegir y ser elegido; sin olvidar la libertad de la individualidad que esto conlleva; fortalecedora de la identidad propia y abierta al diálogo con las otras identidades y culturas; defensora de los derechos humanos no como formulación legal, sino como una apropiación de la razón misma y la naturaleza de toda persona sin distinción de raza, género o ideología; y por otro, como la apropiación de la Constitución Política de Colombia, mediante su conocimiento y respeto; para que de esta manera los ciudadanos que conforman el Estado sean el reflejo de una sociedad más justa e igualitaria; haciendo con esto se debe hacer fuerte y las instituciones y en especial las que imparten justicia.

Ahora, si a estos cambios se les llama desarrollo, se puede decir que las universidades toman importancia en este hecho ya que son las que están entregando a la sociedad los nuevos ciudadanos y lo puede hacer desde dos puntos de vista, a saber: el de convertir al estudiante en una máquina de producción o en un ser capaz de transformarlo todo de una manera crítica y constructiva. Al respecto señala la Unesco «si se carece de instituciones de educación superior y espacios de investigación en ellas adecuados, que formen a una masa crítica de personas cualificadas y cultas, ningún país podrá garantizar un auténtico desarrollo endógeno sostenible» (Unesco, 1998).

Como se ve, ya las entidades internacionales toman partido en ese sentido, haciendo un llamado urgente a las instituciones de educación superior para que transformen sus objetivos, y pasen de ser transmisores del conocimiento a ser parte fundamental del cambio de persona que se debe hacer, teniendo en cuenta para ello estas nuevas exigencias del mundo contemporáneo y con esto la formación de una nueva sociedad. 
Desde luego, los conocimientos son importantes y un buen profesional es pieza clave en una sociedad como la nuestra; pero la parte axiológica desde su proceso de formación no se puede descuidar, por lo que esto constituye una labor fundamental de la educación superior, esto es, formar personas en valores. Al respecto el mismo informe de la Unesco plantea: «La propia educación superior ha de emprender la transformación y las renovaciones más radicales que jamás haya tenido por delante, de forma que la sociedad contemporánea, que en la actualidad vive una profunda crisis de valores pueda trascender las consideraciones meramente económicas y asumir dimensiones de moralidad y espiritualidad más arraigadas» (Unesco, 1998).

¿Cómo se puede lograr esto? Posibilitando espacios educativos que lo fomenten y con profesores que desde sus prácticas pedagógicas lo permitan, promuevan y valoren. Por ello el informe mencionado continua diciendo: «las universidades deben formar a los estudiantes para que se conviertan en ciudadanos bien informados y profundamente motivados, provistos de un sentido crítico y capaces de analizar los problemas de la sociedad, buscar soluciones para los problemas que se planteen en la sociedad, aplicar estas y asumir responsabilidades sociales» (Unesco, 1998).

Como se puede ver, la ciudadanía crítica empieza a emerger como una necesidad de la sociedad actual y obliga a la reflexión si los maestros, por ejemplo, están formados en esa dinámica que permita en el aula la formación de ciudadanos activos, transformadores de su entorno, autónomos, responsables y solidarios, con la capacidad de reflexión y autocrítica para que participen en la transformación de la sociedad hacia una forma más justa, igualitaria y respetuosa. Por esto, Giroux propone que para que las universidades puedan cumplir su papel de transformadoras de personas en dirección de la humanización de la humanidad, el docente debe asumir un papel transformador, lo que significa «ayudar a los estudiantes a adquirir conocimientos críticos acerca de las estructuras básicas de la sociedad, tales como la economía, el Estado, el lugar de trabajo y la cultura de masas» (Giroux, 2006: 146).

Por eso no se puede concebir la transformación de una sociedad sin tener en cuenta al docente, ya que éste debe tener características tales como: conocimiento, compromiso, análisis, síntesis, liderazgo, entre otras habilidades y competencias, para que no solo enseñe, sino también realice la transformación yante todo que pueda también transformarse. En efecto, la pedagogía crítica es la posibilidad de «organizar experiencias pedagógicas dentro de formas y prácticas sociales que aluden al desarrollo de modos más críticos y dialógicos de aprendizaje y lucha» (Giroux, 2006: 192).

Por esta razón los docentes de las universidades tienen que entrar en este rol y para ello deben ser capacitados. Por lo cual las mismas universidades 
deben facilitar este cambio para formar un personal docente actualizado, capaz de generar procesos de transformación, ya que la educación superior ya no es vista como formadora únicamente para el trabajo y el ejercicio disciplinar en el ámbito profesional, sino como una fuente vital para el surgimiento de nuevas generaciones de profesionales diferentes, solidarios, respetuosos, por lo cual «la educación superior tiene que ser defendida como esfera pública vital por derecho propio, o sea, como esfera pública cuyas dimensiones morales y pedagógicas ayudan a renovar la vida cívica» (Giroux, 2003: 361).

Así, lo importante desde la pedagogía crítica es que al estudiante se le debe tratar como persona en el aula de clase; se le debe permitir sentirse libre e identificado; y a la vez, se le deben dar las herramientas necesarias para ser autónomo, al tener la posibilidad de expresar su pensamiento debidamente fundamentado y respetuoso de los demás y por lo tanto debe recibir una formación democrática que lo integre a la sociedad en una forma más provechosa y dinámica, y en la que ésta se vea beneficiada por sus conocimientos y su formación. Al respecto Giroux expone: «Lo que se vuelve medular es que los estudiantes entiendan la forma en que las experiencias de los estudiantes se construyan, a la vez que encaran, porque es gracias a ellas que los estudiantes producen narraciones de quienes son, y se constituyen a sí mismos como individuos particulares... por tanto es imperativo que los maestros y otros educadores aprendan la manera de comprender, legitimar e interrogar tales experiencias...» (2006: 169).

De esta manera, se hace imperativo reconocer la importancia del docente y lo determinante que es hoy día, que éste investigue sobre su quehacer diario, se fundamente, analice, critique y reflexione sobre su contexto social, político económico, y tenga presente el mundo en el que viven y van a vivir sus estudiantes. Así, toda esta comprensión epistemológica del docente debe ser llevada al aula y transmitida a sus estudiantes, para de esta manera se pueda fomentar en ellos dicha actitud y se conviertan en potenciales transformadores sociales planteando soluciones, nuevas y creativas a los problemas de la cotidianidad. Esto es, «lo que los docentes pueden hacer en las aulas es trabajar en sus respectivos roles para desarrollar teorías y métodos pedagógicos que vinculen la auto reflexión y la comprensión a un compromiso de cambiar la naturaleza de la sociedad en general» (Giroux, 2003: 60).

\section{Referencias Bibliográficas:}

Cortina, Adela. 2005. Ciudadanos del mundo. Hacia una teoría de la ciudadanía. Madrid: Alianza Editorial. 
Cortina, Adela. 2007. Ética aplicada y democracia radical. Madrid: Tecnos.

Delors, Jacques. 1966. La educación encierra un tesoro. Madrid: Santillana.

Giroux, Henry. 2006. La escuela y la lucha por la ciudadanía. México: Siglo XXI.

Giroux, Henry. 2003. Pedagogía y política de la esperanza. Traducción de Horacio Pons. Buenos Aires: Amorrortu.

MEN, 2008. Ley 1188, Por la cual se regula el registro calificado de programas de educación superior y se dictan otras disposiciones.

MEN, 2004. Estándares Básicos de Competencias Ciudadanas, Formar para la ciudadanía sí es posible. Ministerio de Educación Nacional de Colombia. http://www. eduteka.org/pdfdir/MENEstandaresCompCiudadanas2004.pdf

MEN. 1994. Ley General de Educación de Colombia.

MEN. 1992. Ley 30, Por la cual se organiza el servicio público de la Educación Superior.

Mockus, Antanas. 2004. «¿Por qué competencias ciudadanas en Colombia?». Al Tablero, http://www.mineducacion.gov.co/1621/article-87299.html

Ortega y Gasset, José. 1963. Misión de la Universidad. Montevideo: Gallo.

Martínez, Jaume (coord.). 2003. Ciudadanía, poder y educación. Barcelona: Ed. Graó.

Larrain U., Ana María y Luis Eduardo González. s.f. «Formación Universitaria por Competencias». http://sicevaes.csuca.org/attachments/134_Formacion\%20Universitaria\%20por\%20 competencias.PDF

Martínez Martin, Miguel. 2006. «Formación para la ciudadanía y la educación superior». Revista Iberoamerica de Educación. 42: 85-102.

Pinto Cueto, Luisa, 1999. "Currículo por competencias: Necesidad de una Nueva Escuela». Tarea 43: 10-17.

Platón. s.f. La República. En: http://www.bibliotecasvirtuales.com/biblioteca/ otrosautoresdelaliteraturauniversal/Platon/larepublica/index.asp. (Consultado el 16 de septiembre de 2010).

Rozo Acuña, Eduardo. 1987. Evolución de las ideas políticas. Bogotá: Ed. Universidad Externado de Colombia.

Ruiz, Alexander y Enrique Chaux, 2001. La formación en competencias ciudadanas. Bogotá: Ascofade. En: http://www.redes-cepalcala.org/inspector/DOCUMENTOS\%20 


\section{Revista de}

investigaciones UNAD

Volumen 11. Número 1. Junio 2012

\section{Y\%2OLIBROS/COMPETENCIAS/LA\%2OFORMACION\%20DE\%20COMPETENCIAS\%20 CIUDADANAS.pdf}

Unesco. 1998. Conferencia mundial sobre la educación superior. En: http://www. unesco.org/education/educprog/wche/declaration_spa.htm 